\title{
The Effects of Long-term Exercise on Cerebral Function and the Maintenance of Concentration in the Elderly
}

\author{
Shukoh Haga ${ }^{1}$, Takuya Sakurai ${ }^{2 *}$, Shogo Sato $^{2}$, Michiko Sasahara $^{3}$, Fumio Aita ${ }^{4}$, Kazuki Esaki ${ }^{5}$, \\ Koji Toshinai ${ }^{6}$, Etsuo Ueya ${ }^{7}$, Noboru Hashimoto ${ }^{8}$, Junetsu Ogasawara ${ }^{2}$,Takako Kizaki ${ }^{2}$, Yoshinaga \\ Ishibashi ${ }^{2}$,Tomonobu Sakurai ${ }^{9}$, Shuji Oh-ishi ${ }^{10}$, Hideki Ohno ${ }^{2}$ and Eimatsu Takakuwa ${ }^{11}$ \\ ${ }^{1}$ Professor Emeritus, Institute of Health Sport Science, University of Tsukuba, Tsukuba, Ibaraki, Japan \\ ${ }^{2}$ Department of Molecular Predictive Medicine and Sport Science, Kyorin University, School of Medicine, Japan \\ ${ }^{3}$ Sasahara Fitness Planning, Japan \\ ${ }^{4}$ Edosaki Sohgoh High School, Japan \\ ${ }^{5}$ Faculty of Education, Shiga University, Japan \\ ${ }^{6}$ Neurology, Respirology, Endocrinology, and Metabolism, Department of Internal Medicine, Faculty of Medicine, University of Miyazaki, Japan \\ ${ }^{7}$ Faculty of Comprehensive Welfare, Department of Comprehensive Welfare, Urawa University, Japan \\ ${ }^{8}$ Department of Judo Therapy, Faculty of Health Sciences, Tokyo Ariake University of Medical and Health Sciences, Japan \\ ${ }^{9}$ Faculty of Bio-Industry, Tokyo University of Agriculture, Japan \\ ${ }^{10}$ Department of Respiratory Medicine, Hachioji Medical Center, Tokyo Medical University, Japan \\ ${ }^{11}$ Professor Emeritus, Department of Hygiene and Preventive Medicine, Hokkaido University, School of Medicine, Japan
}

Received: November 26, 2013; Accepted: December 27, 2013; Published: December 31, 2013

“Corresponding author: Takuya Sakurai, Kyorin University, School of Medicine, Japan; Tel: +81 42247 5511; Fax: +81 422444427 ; E-mail: sakutaku@ ks.kyorin-u.ac.jp

\begin{abstract}
The purpose of this study was to clarify the effect of long-term exercise on the maintenance of concentration, as an indicator of the activity level of the cerebral cortex, and on the Profile of Mood States (POMS) in healthy elderly persons. Forty-six subjects were divided into three groups: a long-term exercise group (LTE-G) of 21 subjects (mean age: 67 years); a health exercise group (HE-G) of 12 subjects (mean age: 69 years); and a sedentary control group (SC-G) of 13 subjects (mean age: 70 years). We assessed the target aiming function (TAF), the POMS, and the presence in the urine of the following compounds: 8-hydroxydeoxyguanosine (8-OHdG) and acrolein (ACR) as indicators of oxidative stress; adrenaline, noradrenaline, and dopamine as indicators of sympathetic nervous activity. The mean TAF-L values, which represent the level of mental concentration, were statistically lower in the LTE-G and HE-G than in the SC-G. The mean TAF-D values, indicating deviation levels, were significantly lower in the HE-G than in the SC-G. Total Mood Disturbance (TMD) scores, which are the scored totals of POMS tests, and the scores on the "Confusion" factor were clearly lower in the LTE-G and HE-G than in the SC-G. On the other hand, there were no significant differences in 8-OHdG, ACR, adrenaline, noradrenaline or dopamine values among the three groups. These results suggest that the continuation of exercise improves the cerebral cortical activity, the maintenance of concentration, and the POMS scores of healthy elderly persons.
\end{abstract}

Keywords: Healthy exercise; Long-term exercise; Maintenance of concentration; Cerebral cortical activity; Profile of Mood States

\section{Introduction}

Elderly population in Japan has risen in recent years, by about 23.3\% in 2011 (Cabinet Office, Annual Report on the Aging Society), and at the same time the average life expectancy has extended to about 80 years for men and 86 years for women, which has resulted in a type of aging society never seen before. Medical care and welfare have, therefore, become important issues for the maintenance of healthy life expectancy in the age of decline. Aging is normally accelerated in the age of decline, but regular exercise is an anti-aging method that improves health.

Exercise has been shown to be beneficial in the elderly, improving bodily functions such as $\mathrm{VO}_{2}$ max and the respiratory system [1,2], the circulatory system. [1-7], metabolic hormones $[8,9]$, muscular hypertrophy $[9,10]$, the immune system [11$13]$, and antioxidant enzymes $[14,15]$. Exercise also produces a positive effect on the psychological and emotional state and alleviates stress [16]. In order to assist elderly people in carrying on healthy, independent social lives, it would be helpful to know whether the plasticity of the brain is enhanced by the maintenance of exercise and sports activities, and how it relates to activity level and sustainability.

The cerebrum's function in maintaining concentration [target aiming function (TAF)] has been defined and developed by Takakuwa [17]. TAF is the ability to maintain mental concentration by maintaining a high level of cerebral cortical activity, and represents the integrated results of the cerebrum's mental and physiological functions. The TAF test is an objective, numerical method for the measurement that indicates how well cerebral cortical activity level is maintained, and it makes statistical analysis possible. Previous studies have reported that advanced marksmen, pilots, and trained athletes can maintain high levels of concentration evaluated by the TAF test $[18,19]$. 
Nevertheless, there has been little information available about the effect of physical exercise on the levels of mental concentration in elderly people, whereas many reports state that physical exercise can prevent and improve memory impairment in adult humans [20-22].

Thus, the aim of the present study was to examine the effects of continuous exercise on cerebral cortical activity for the maintenance of concentration in the elderly, in conjunction with oxidative stress indicators such as 8-hydroxydeoxyguanosine (8OHdG) [23] and acrolein (ACR) [24], and sympathetic nervous activity indicators like adrenaline, noradrenaline and dopamine concentrations, as well as assessment with the Profile of Mood States (POMS).

\section{Materials and Methods}

\section{Subjects}

The subjects in the long-term exercise group (LTE-G) were 21 elderly people ( 4 males, 17 females) who had played tennis, run or jogged for 20 to 40 years, and were currently continuing these activities 2 to 5 times a week. The subjects in the health exercise group (HE-G) were 12 elderly people (5 males, 7 females) who had been participating in health exercise clubs once a week for 90 minutes for 4 years, and did daily activities such as integrated compound movements and walking. We also examined 13 elderly people (4 males, 9 females), who engaged in no type of exercise and led a sedentary life, as the sedentary control group (SC-G).

We explained the aim of the research to the subjects and obtained their written informed consent. Experimental procedures were approved by the Ethics Committee of the Institute of Health and Sport Science, University of Tsukuba, Japan.

\section{Body and blood pressure measurements}

After conducting interviews and health examinations, we measured the subjects' height and weight to determine their body mass index (BMI). Blood pressure was measured by the auscultatory method using the Manchette method's mercury manometer after subjects rested sufficiently in a sitting position. We also measured the pulse rateat rest by palpating the radial artery. We then measured the body fat percentage using the Impedance method and aHDF-302 body fat scale made by Omron (Kyoto, Japan). Physical characteristics of the three groups are shown in Table 1.

\section{Measuring the maintenance of concentration}

The maintenance of concentration was measured using the TAF test developed by Takakuwa [17] (Figure 1). The measurement is taken by asking the subjects to peep at a device through their dominant eye, and aim for a target emitting a 2.5 $\mathrm{cm}$ diameter circle of light with their dominant arm continuously for 1 minute, continuing in the same manner for a total of 3 minutes with 10 second breaks after each minute. The temporal variation accuracy was recorded as a continuous curve, and was simultaneously evaluated by converting the result to a numeral. For the evaluation, the top line of the recording sheet was marked 0.0 , and it went up to a score of 10.0 (the bottom line). A mark was plotted every 2.5 seconds, and the average scores for each minute

\begin{tabular}{|c|c|c|c|}
\hline & LTE-G (n=21) & HE-G (n=12) & SC-G (n=13) \\
\hline Age (yr) & $66.6 \pm 4.0^{*}$ & $68.6 \pm 5.4$ & $70.3 \pm 5.6$ \\
\hline Height (cm) & $161.9 \pm 5.9^{\#}$ & $160.7 \pm 5.8$ & $154.5 \pm 7.4$ \\
\hline Weight $(\mathrm{kg})$ & $58.9 \pm 7.8$ & $61.2 \pm 5.7^{*}$ & $52.8 \pm 8.8$ \\
\hline BMI $\left(\mathrm{kg} / \mathrm{m}^{2}\right)$ & $22.4 \pm 2.1$ & $23.7 \pm 2.7$ & $22.3 \pm 3.6$ \\
\hline \%Fat $(\%)$ & $27.5 \pm 4.9$ & $27.3 \pm 6.6$ & $29.6 \pm 5.7$ \\
\hline PR (beats/min) & $62.1 \pm 6.2^{\#} \$$ & $70.1 \pm 7.4$ & $69.8 \pm 7.7$ \\
\hline SBP (mmHg) & $136.1 \pm 19.8$ & $132.9 \pm 17.5$ & $128.4 \pm 11.5$ \\
\hline DBP (mmHg) & $82.5 \pm 10.6^{\#}$ & $75.1 \pm 7.4$ & $72.0 \pm 8.2$ \\
\hline
\end{tabular}

Values are means \pm SD.

*Significant difference from SC-G $(\mathrm{p}<0.05)$

\#Significant difference from SC-G $(\mathrm{p}<0.01)$

\$Significant difference from HE-G $(\mathrm{p}<0.01)$

Table1: Physical characteristics of three groups.

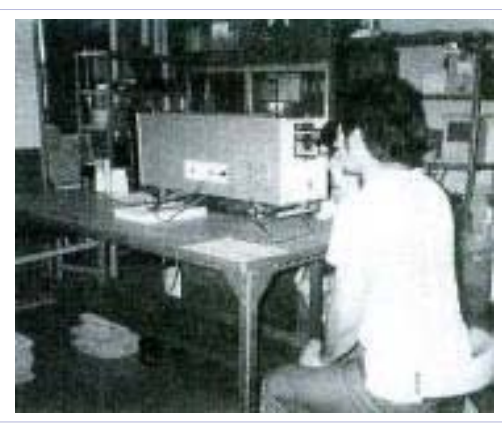

Figure 1: The picture of TAF test.

were designated as L1, L2 and L3. The average score of the three results was designated as TAF-L, and the standard deviation was designated as TAF-D, respectively. TAF-L represented the level of mental concentration, and TAF-D showed the fluctuation in the mental concentration time. The lower the score falls, the higher the maintenance of concentration rises. It should be noted that this measurement was carried out after explanation of the measurement process, and 1 minute of practice.

\section{Mood profile examination}

We evaluated the moods and emotions of subjects over the past week using the POMS [25], which is a questionnaire method used to evaluate 6 mood scales. The scales [Tension], [Depression], [Anger], [Vigor], [Fatigue], and [Confusion], were all evaluated, and we drew a total vigor score from the total assembled score of the scales, excluding [Vigor]. The Total Mood Disturbance (TMD) score was calculated by adding 100 points, and the group scores were compared.

\section{Measuring urine oxidative stress markers and catecholamines}

After about $25 \mathrm{ml}$ of urine was collected in a measuring cup, $1 \mathrm{ml}$ of urine was placed in each of 4 micro-tubes and preserved at $-80^{\circ} \mathrm{C}$. A few days later, the concentrations of $8-\mathrm{OHdG}$, $\mathrm{ACR}$, adrenaline, noradrenaline, and dopamine in urine were measured using the methods described as follows: We used an 8-OHdG Check ELISA (Japan Institute for the Control of Aging, Shizuoka, Japan) to measure 8-OHdG, and used an ACR ELISA kit (NOF Corporation, Tokyo, Japan) to measure ACR concentration. Next, conventional reverse-phase high performance liquid chromatography was performed by the SRL testing center 
(Tachikawa, Japan) to measure adrenaline, noradrenaline and dopamine concentrations.

\section{Statistical analysis}

The numbers shown in the table and figures are the average score \pm the standard deviation (SD). After the Bartlett test was carried out, statistical analysis of the measurement results was performed using one-factor ANOVA and the Kurskal-Wallis test. The test of the differences between the groups was performed using the post-hoc test (Tukey-Kramer method), and levels of significance were set at $\mathrm{p}<0.05$.

\section{Results}

\section{Body measurements}

The three groups' physical characteristics are shown in Table 1. The age of the LTE-G was significantly lower $(p<0.05)$ than that of the SC-G. In terms of physical characteristics, the height of the LTE-G was significantly higher $(\mathrm{p}<0.01)$, and the weight score of the HE-G was significantly higher $(p<0.05)$ than those of the SC-G, respectively. The BMI was in the range of 22 to 24 for all three groups, and the $\%$ fat was about $27-30 \%$. Thus, no significant differences in BMI or \% fat was observed among the three groups. The systolic blood pressure (SBP) was 128-136 $\mathrm{mmHg}$, and there were no significant differences among the three groups, which appeared to be all within normal range. On the other hand, the diastolic blood pressure (DBP) of the LTE-G was significantly higher $(p<0.01)$ than that of the SC-G, but all three groups were within the normal range of $72-83 \mathrm{mmHg}$. The pulse rate at rest was 62 beats/min in the LTE-G, which was a significantly lower $(p<0.01)$ score than that of either the HE-G or the SC-G.

\section{Maintenance of concentration}

The TAF-L showing the maintenance of concentration for both the LTE-G and the HE-G, had a score that was significantly lower $(p<0.0001)$ than that of the SC-G, indicating excellent concentration maintenance for the LTE-G and for the HE-G (Figure 2A). An exercise activity group (EA-G), made up of LTE-G and HE-G, was divided by age into a 60's group and a 70's group, and each age group was compared with its equivalent from the SC-G (Figure 2B). For both age groups, the maintenance of concentration score was significantly lower $(\mathrm{p}<0.01, \mathrm{p}<$ 0.05 ) for the EA-G, which indicated excellent maintenance of concentration for this group (Figure 2B). When the TAF-D of the EA-G, which shows fluctuation during the maintenance of concentration measurement, was compared with that of the SCG, no significant differences were observed with LTE-G, but the TAF-D score was significantly lower $(p<0.01)$ for HE-G (Figure 3).

\section{8-OHdG, ACR, adrenaline, noradrenaline and dopamine concentrations}

No significant differences were observed among the three groups in terms of 8-OHdG, ACR, adrenaline, noradrenaline or dopamine concentration in urine (data not shown).

\section{Mood profile examinations}

There were no significant differences in Tension, Depression,
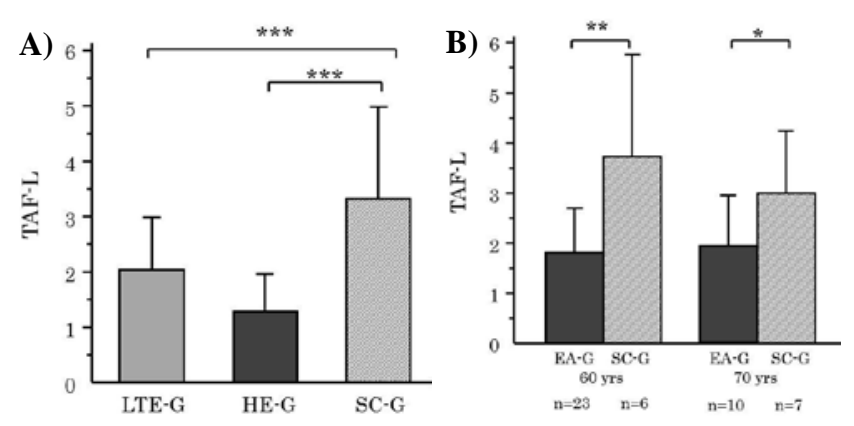

Figure 2: The effects of long-term exercise on maintenance of concentration in elderly people.

The maintenance of concentration function for the long-term exercise group (LTE-G), health exercise group (HE-G) and sedentary control group (SC-G) was measured using the target aiming function (TAF) test, and the TAF-L score shown is the average of the 3 results.

(A) TAF-L comparison among LTE-G, HE-G and SC-G. The mean \pm SD (LTE-G: $\mathrm{n}=21$; HE-G: $\mathrm{n}=12$; $\mathrm{SC}-\mathrm{G}: \mathrm{n}=13$ ) is given.

${ }^{* * *}$ Significant difference from $\mathrm{SC}-\mathrm{G}(\mathrm{p}<0.0001)$.

(B) The relationship between exercise habits and TAF-L by age group. LTE-G and HE-G were combined to form an exercise activity group (EAG), which was then divided by age into subjects in their 60's and 70's, and compared with their equivalent age groups in SC-G. The mean $\pm S D$ [EA-G (60 yrs): $\mathrm{n}=23$; EA-G (70 yrs): $\mathrm{n}=10$; SC-G $(60 \mathrm{yrs}): \mathrm{n}=6$; SC-G ( $70 \mathrm{yrs}): \mathrm{n}=7$ ] is given. ${ }^{*}$ Significant difference from $S C-G(p<0.05)$. ${ }^{* *}$ Significant difference from SC-G $(\mathrm{p}<0.01)$.

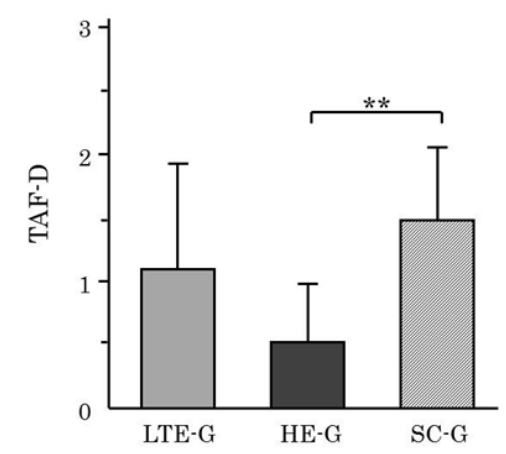

Figure 3: The effects of long-term exercise on fluctuation in maintenance of concentration.

The standard deviation of TAF-L was calculated as TAF-D, then compared among LTE-G, HE- G and SC-G. TAF-D shows the fluctuation in the mental concentration time. The mean \pm SD (LTG-G: $n=21$; HE-G: $n=12$; SC-G: $\mathrm{n}=13$ ) is given.

${ }^{* *}$ Significant difference from SC-G $(\mathrm{p}<0.01)$.

Anger, Vigor or Fatigue, but the LTE-G and HE-G had significantly lower values $(p<0.05)$ for Confusion compared to the SC-G (Figure 4). The LTE-G also had a significantly lower TMD score (p $<0.05$ ) compared with that of the SC-G (Figure 5).

\section{Discussion}

The ages of the subjects were significantly lower in the LTE-G compared to the SC-G, but since subjects in both LTE-G and SC-G were in the early age of decline of 65 to 70 , they were considered over all to be more or less the same age. With respect to physical characteristics, LTE-G and HE-G scored higher for height and 
weight, respectively, in comparison to the SC-G, but the BMI score was almost the same for all the groups, falling within the normal range. The SBP score of the three groups was within the normal range of approximately $130 \mathrm{mmHg}$, and DBP was around 70 to 80 $\mathrm{mmHg}$, also within the normal range. Therefore, all three groups were speculated to be in a healthy physical condition. Meanwhile, the resting pulse rate score of LTE- $G$ was significantly lower than that of the SC-G. This is likely due because endurance exercise had been continued over a very long period of 20 to 40 years, leading to an increase in chronic vein reflux during exercise, an enlargement of the left ventricular end-diastolic diameter and an increase in the left ventricular volume, which in turn resulted in an increase in myocardial contractile force. As a result, the heartbeat output increased $[7,26]$, and at the same time produced

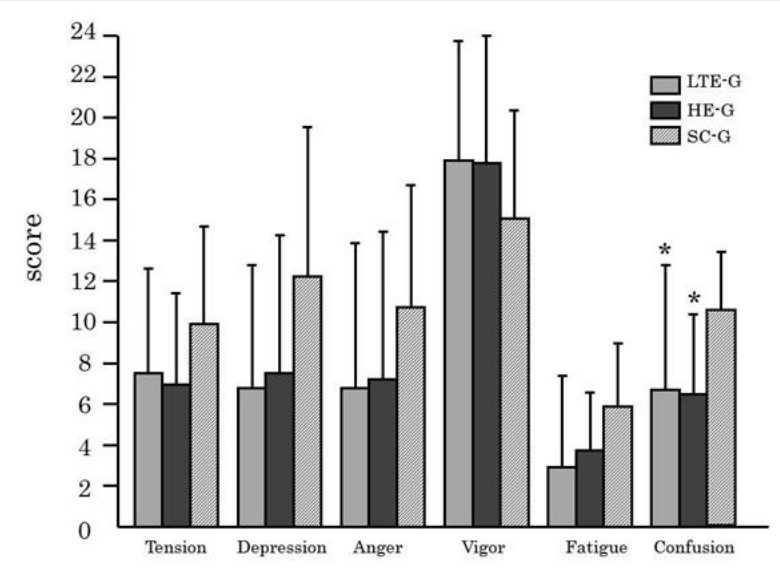

Figure 4: The effects of long-term exercise on the mood and emotional state in elderly people.

We evaluated the mood and emotional state of subjects in all the groups, dating back one week, using the Profile of Mood States (POMS). The mean \pm SD (LTG-G: $n=21$; HE-G: $n=12$; SC- $\mathrm{a}: \mathrm{n}=13$ ) is given. *Significant difference from SC-G $(p<0.05)$.

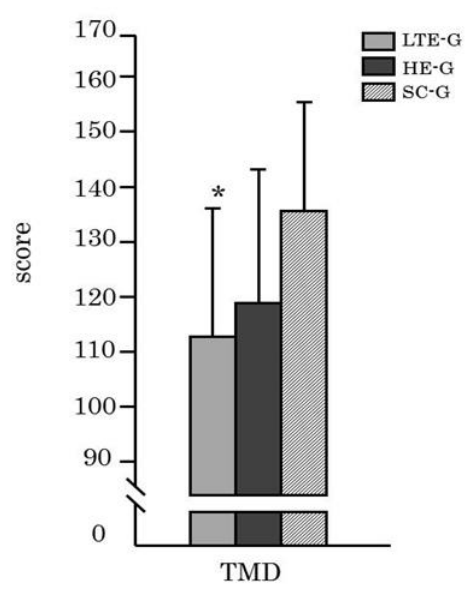

Figure 5: The effects of long-term exercise on the Total Mood Disturbance (TMD) in elderly people.

We calculated the overall affective index (TMD) using the POMS results as a basis, and compared this among the groups. The mean \pm SD (LTG-G: $\mathrm{n}=21$; HE-G: $\mathrm{n}=12$; SC-G: $\mathrm{n}=13$ ) is given.

*Significant difference from SC-G $(\mathrm{p}<0.05)$. the effect to bradycardia at rest due to vagal tone increase, reflecting compensation for the lower resting pulse rate.

Normally, the form and function of neurons and the neural network in the brain change in response to environmental factors, but they usually mature through the growth and developmental stages, and then, as people enter middle and old age, learning ability and memory are thought to decline [27]. Many epidemiological studies, intervention trials, and animal experiments have demonstrated that memory dysfunction, which is the predominant symptom of dementia and Alzheimer's disease, can be prevented and reduced in aged subjects through physical exercise [20-22]. However, the effects of physical exercise on mental concentration in the elderly are still unclear.

In this research we found that the maintenance of concentration in LTE-G and HE-G was clearly superior to that of SC-G. Even when examined by age, the results were almost the same for those in their 60's and 70's. The fluctuation in the maintenance of concentration (TAF-D) was also significantly smaller in HE-G compared to the SC-G, suggesting that the subjects were able to focus their concentration on one point in a relaxed and calmemotional state during the test. These results show that a high level of cerebral cortex activity and concentration can be maintained, even if the duration or amount of exercise differs.

In elderly people who have led an in active life style, the effects of aging are greater, and the onsets of developing cerebrovascular ischemia are higher. In fact, a decline in cerebral blood flow is the basis behind the onset of vascular dementia, and a strong correlation has been reported between the amount of decreased blood flow and the progression of dementia [28]. However, in a report [29] related to exercise and cerebral blood flow, when an exercise pushing a spring with the thumb and index finger together was carried out, the local blood flow of the primary motor cortex tended to increase linearly in proportion to the frequency of the spring exercise, compared to the resting state. Furthermore, exercising the entire body using an incremental load method notably increases the blood volume in the brain to the level of $50-60 \%$ of $\mathrm{VO}_{2}$ max [30]. Even 30 seconds of anaerobic exercise increases the oxygen level and blood volume in the brain [31]. The loss of neurons due to aging is an inevitable phenomenon, but it has been suggested that the dentate gyrus nerves in the hippocampus of humans are regenerated after maturation [32]. It is known that exercise increases neurogenesis in the dentate gyrus [33]. When information input into the brain is determined by the amygdala to be either "pleasant" or "valued" the activity of the entire brain is enhanced via the humoral regulation system, which improves learning in the cerebral cortex, and by building the neural network [34], stimulates "the perceptions of pleasant" and "cognitive functions of value" enhancing the brain's internal activity level.

The results of this study, taken together, suggest that when elderly people continue to enjoy moderate exercise 1-3 times a week over a long period of time, exercise contributes greatly to learning ability and memory, which are thought to decline when the brain ages, and also contribute to the maintenance and improvement of the cerebral cortical activity level and maintenance of concentration. This desirable phenomenon occurs not only in persons in their 60's, but also in those in their 
70 's. Nevertheless, the results from the present study seem to causative because we did not actually perform fixed exercise to the elderly subjects. Therefore, there is no denying that other factor(s) is involved in the maintenance of concentration observed in exercise group, and further studies are required.

Oxidative stress is thought to be connected to enhanced aging and illness [35]. In this research, we examined the effects on oxidative stress indicators and sympathetic nervous reaction indicators from the viewpoint of aging and exercise. We saw no differences between any of the measured scores of the three groups, thereby suggesting that oxidative stress level in healthy persons does not affect the brain's concentration maintenance function.

With regard to the psychological effects of exercise, according to the International Society of Sport Psychology, exercise creates an emotional effect, regardless of sex or age, by decreasing anxiety, depression and various stress indicators [36](International Society of Sport Psychology). This study used the POMS to examine the effect of continuous exercise on mood in daily life. It was found that LTE-G and HE-G scored significantly lower on the [Confusion] scale compared to SC-G. LTE-G also had a lower overall TMD score on the 6 POMS scales in comparison to SC-G, clearly indicating a stable mood and suggesting that continuous exercise contributes to the enhancement of mental self-control even in aged persons. Exercise requires the participant to always stay relaxed and calm, and to respond quickly. While playing tennis, instant response is required to the ball's movements and developments in the game, and in the case of running or jogging it is essential to pay attention to the speed, level of fatigue and physical condition, and the like. It is also important that those who participate in health exercise classes respond correctly to the instructor's explanations and important points, and that they are required to learn these things. When exercise becomes habitual, the [Confusion] scale will naturally be decreased in daily life, improving emotional stability and calmness. In previous studies it has become clear that transient exercise has antidepressant and anxiolytic effects, [37] and that exercise contributes to the reduction of anxiety and an increase in positive feelings [38,39] Improving one's mood creates mental stability, resulting in an enhancement of the cerebralcortical activity level, and is thought to contribute to the maintenance of concentration fluctuation.

These results, taken together, indicate that if elderly people habitually continue to exercise, it enhances their cerebral activity level and maintenance of concentration, resulting in a more stable mood.

\section{Acknowledgement}

We would like to thank THE DESCENTE AND ISHIMOTO MEMORIAL FOUNDATION FOR THE PROMOTION OF SPORTS SCIENCE for the grant that they generously awarded to this research. This work was also supported by Grants in Aid for Specific Project Research from the Ministry of Education, Culture, Sports, Science and Technology of Japan.

\section{References}

1. Seals DR, Hagberg JM, Hurley BF, Ehsani AA, et al. (1984) Endurance training in oldermen and women. I. Cardiovascular responses to exercise. J Appl Physiol 57(4): 1024-1029.
2. Haga S (1990) Train ability of aerobic exercise. Shinkoh-KohekiMedical Publish, Tokyo, 74-148.

3. De Vries HA (1974) Physiological effect of an exercise training regimen upon men aged 52-88. The Journal of Gerontology 25(4): 325-336.

4. Fleg JL, Schulman SP, O'connor, FC, Gerstenblith G, et al. (1994) Cardiovascular responses to exhaustive upright cycle exercise in highly trained older men. J Appl Physiol 77(3): 1500-1506.

5. Hagberg JM, Montain SJ, Martin WH, Ehsani AA (1989) Effect of exercise training in60-to 69-year-old persons with essential hypertension. The American Journal of Cardiology 64(4): 348-353.

6. Levy WC, Cerqueira MD, Abrass IB, Schwartz RS, et al. (1993) Endurance exercise training augments diastolicfilling atrestand during exercise in healthy young and oldermen. Circulation 88(1): 116-126.

7. Seals DR, Hagberg JM, Spina RJ, Rogers MA, et al. (1994) Enhanced left ventricular performance in endurance trained older men. Circulation 89(1): 198-205.

8. Torino R (1989) Effects of physical training on the insulin resistance of aging. Am J Physiol Endocrinol Metab 256(3 Pt 1): E352-E356.

9. Frontera WR, Meredith CN, O'reilly KP, Knuttgen HG, et al. (1985) Strength conditioning in older men: skeletal muscle hypertrophy and improved function. J ApplPhysiol 64: 1038-1044.

10. Pyka G, Lindenberger E, Charette S, Marcus R (1994) Muscle strength and fiber adaptations to a year-long resistance training program in elderly men and women. J Gerontol 49(1): M22-M27.

11. Crist DM, Mackinnon LT, Thompson RF, Atterbom HA, et al. (1989) Physical exercise increases natural cellular mediated tumorcytotoxicity in elderly women. Gerontology, 35(2-3): 66-71.

12. Okutsu M, Yoshida Y, Zhang X, Tamagawa A, et al. (2008) Exercise training enhances in vivo tuberculosis purified protein derivative response in the elderly. J Appl Physiol 104(6): 1690-1696.

13. Shephara RJ, Shek PN (1995) Exercise, aging and immune function. Int J Sports Med 16(1): 1-6.

14. Pedersen BK, Febbraio MA (2008) Muscleas an endocrine organ: focus on muscle-derived interleukin-6. Physiological Reviews 8(4): 13791406.

15. Walther C, Gielen S, Hambrecht R (2004) The effect of exercise training on endothelial function in cardiovascular disease in humans. Exerc Sport Sci Rev 32(4): 129-134.

16. Moses J, Steptone A, Mathews A, Edwards S (1989) The effects of exercise training on mental well-being in the normal population: a controlled trial. J Psy chosom Res 33(1): 47-61.

17. Takakuwa E (1971) Maintaining concentration (TAF) as a measure of mental stress. Ergonomics 14(1): 145-158.

18. Miyazaki T, Takakuwa E, Koizumi K (1963) Study based on the original method on measurement of function of concentration maintenance (target aiming function) as a physical fitness test. The National Defense University 10(11): 513-515.

19. Takakuwa E (1980) Study on fatigue-focus on the target aiming function (TAF). Jpn J Hyg 35(1): 78-85.

20. Archer T (2011) Physical exercise alleviates debilities of normal aging and Alzheimer's disease. Acta Neurologica Scandinavica 123(4): 221238.

21. Hillman $\mathrm{CH}$, Erickson KI, Kramer AF (2008) Be smart, exercise 
your heart: exercise effects on brain and cognition. Nature Reviews Neuroscience 9 (1): 58-65.

22.vanPraag $H$ (2009) Exercise and the brain: something to chew on Trends Neurosci 32(5): 283-290.

23. Okamura K, Doi T, Hamada K, Sakurai M, et al. (1997). Effect of repeated exercise on urinary 8-hydroxy-deoxyguanosine excretion in humans. Free Radical Research 26(6): 507-514.

24. Uchida K, Kanematsu M, Sakai K, Matsuda T, et al. (1998) Proteinbound acrolein: potential markers for oxidative stress. Proc Natl Acad Sci USA 95(9): 4882-4887.

25. Yokoyama K, Araki S, Kawakami N, Takeshita T (1990) Production of the Japanese edition of profile of mood states (POMS): assessment of reliability and validity. Nihon Koshu Eisei Zasshi 37(11): 913-918.

26. Levy WC, Cerqueira MD, Abrass IB, Schwartz RS, et al. (1993) Endurance exercise training augments diastolicfilling atrestand during exercise in healthy young and oldermen. Circulation 88(1): 116-126.

27. Hatanaka H, Ikegami S, Arimatsu Y (1994) Aging of brain-study between life and death of neuron. Brain science series 2. Tokyo: Kyoritsu publish, 2-16.

28. Matsubayashi K, Matsumoto M, Kawamoto A, Shimada K, et al. (1988) Evaluation of the cerebral lesion and perfusion as risk factors in vascular dementia. Jpn J Geriatrics 25(6): 569-575.

29. Kawashima R, Okuda K, Fukuda H (1996) Blood circulation on the brain for the performance of physical exercise. J Health Phys Educ Recreatio 46(10): 801-805.

30. Yasuchochi M, Be M, Sasaki M, Koseki S, et al. (1996) Dynamic movement in cerebral oxygen saturation and blood volume by nearinfrared spectroscopy. Jpn J Phys Fit Sports Med 45(6): 686.
31. Yasuchochi M, Bae SY, Koseki S, Nakase Y, et al. (1997) Changes of regional cerebral blood volume and oxygenation during anaerobic exercise and recover. Ther Res 18(7): 2340-2345.

32. Eriksson PS, Perfilieva E, Bjork-Eriksson T, Alborn AM, Nordborg C, et al. (1998) Neurogenesis in the adult human hippocampus. Nature Medicine 4(11): 1313-1317.

33.vanPraag H, Christie BR, Sejnowski TJ, Gage FH (1999) Running enhances neurogenesis, learning, and long-term potentiation in mice. Proc Natl Acad Sci USA 96(23): 13427-13431.

34. Matsumoto G (1995) Emotion elucidated through functional reconstitution of the brain. Brain Sci Med Disorders 6(4): 365-382.

35.Suzuki K, Ohno H, Oh-ishi S, Kizaki T, et al. (1994) Superoxide dismutases in exercise and disease. In Sen CK, Packer L \& Hanninen 0 (Eds.), Exercise and Oxygen Toxicity. Elsevier, Amsterdam, 127-161.

36. International Society of Sport Psychology (1992) Physical activity and Psychological benefits. A position statement from the international society of sport psychology. Journal of Applied Sport Psychology 4(1): 94-98.

37. Dishman RK (1997) Brain monoamines, exercise, and behavioral stress: animal models. Med Sci Sports Exerc 29(1): 63-74.

38. Petruzzello SJ, Landers DM (1994) State anxiety reduction and exercise: does hemispheric activation reflect such changes. Medicine and Science in Sports and Exercise's 26(8): 1028-1035.

39. Petruzzello SJ, Tate AK (1997) Brain activation, affect, and aerobic exercise: an examination of both state-independent and statedependent relationships. Psychophysiology 34(5): 527-533. 